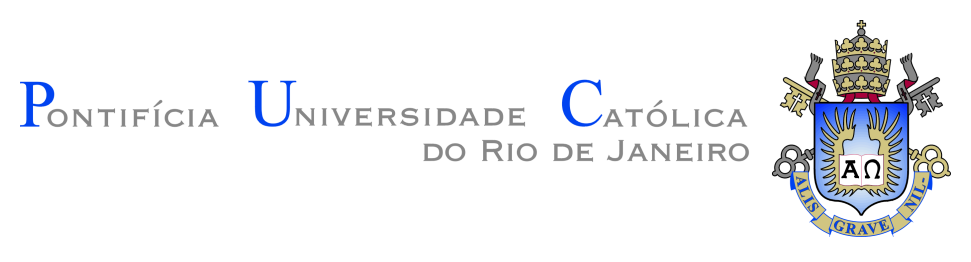

Percy Wilianson Lovon Ramos

\title{
Modeling and Control Design of a Tracked Mobile Robot for Surveillance Tasks
}

Dissertação de Mestrado

Dissertation presented to the Programa de Pós-graduação em Engenharia Elétrica da PUC-Rio in partial fulfillment of the requirements for the degree of Mestre em Engenharia Elétrica

Advisor: Prof. Antonio Candea Leite 
Percy Wilianson Lovon Ramos

\section{Modeling and Control Design of a Tracked Mobile Robot for Surveillance Tasks}

Dissertation presented to the Programa de Pós-graduação em Engenharia Elétrica da PUC-Rio in partial fulfillment of the requirements for the degree of Mestre em Engenharia Elétrica . Approved by the Examination Committee.

Prof. Antonio Candea Leite

Advisor

Departamento de Engenharia Elétrica - PUC-Rio

Prof. Paulo César Pellanda Instituto Militar de Engenharia - IME

Prof. Ramon Romankevicius Costa Universidade Federal do Rio de Janeiro - UFRJ

Prof. José Paulo Vilela Soares da Cunha Universidade do Estado do Rio de Janeiro - UERJ 
All rights reserved.

\section{Percy Wilianson Lovon Ramos}

Systems Engineering by National San Agustin University (Arequipa, Perú)

Bibliographic data

Lovon Ramos, Percy Wilianson

Modeling and Control Design of a Tracked Mobile Robot for Surveillance Tasks / Percy Wilianson Lovon Ramos; advisor: Antonio Candea Leite, - 2019.

v., 118 f: il. color. ; $30 \mathrm{~cm}$

Dissertação (mestrado) - Pontifícia Universidade Católica do Rio de Janeiro, Departamento de Engenharia Elétrica, 2019.

Inclui bibliografia

1. Engenharia Elétrica - Teses. 2. Robôs Móveis com Esteiras;. 3. Controle Robusto;. 4. Sistemas Incertos;. 5. Interface de Usuário Móvel;. 6. Robôs de Vigilância;. I. Leite, Antonio Candea. II. Pontifícia Universidade Católica do Rio de Janeiro. Departamento de Engenharia Elétrica. III. Título. 

and encouragement. 


\section{Acknowledgments}

I would like to first thank my parents for all the support during the development of this thesis. I dedicate all my gratitude, to my sister and my brother for being there to remind me the correct path, and my beloved nephews.

I would like to thank my friends that I met during the process of development this thesis and my colleagues from the Intelligent Control Laboratory (LCI), for all the experiences during this time, I always will be grateful with you guys. I would like to thank my advisor, Professor Antonio C. Leite, for all the technical teaching, advices and personal lessons learned during this time, for always be there when I have a doubt or problem.

This study was financed in part by the Coordenação de Aperfeiçoamento de Pessoal de Nível Superior - Brasil (CAPES) - Finance Code 001. 


\section{Abstract}

Lovon Ramos, Percy Wilianson; Leite, Antonio Candea (Advisor). Modeling and Control Design of a Tracked Mobile Robot for Surveillance Tasks. Rio de Janeiro, 2019. 118p. Dissertação de Mestrado - Departamento de Engenharia Elétrica, Pontifícia Universidade Católica do Rio de Janeiro.

In recent years, the latest advances in robotics and its applications have been used to reduce the workload and manpower requirements, improving the environment, health and safety (EHS) conditions, particularly in agricultural production and farming systems. Autonomous robots are part of such technological innovation and Tracked Mobile Robots (TMRs), in particular, have being widely used on agricultural fields around the world, since their tracks provide a large contact area on the wet soils and irregular terrains avoiding the robot to get stuck. In this work, we address the modeling and control design of tracked mobile robots (TMRs) able to perform surveillance tasks in agricultural fields. The proposed methodology considers that the kinematic models of the TMRs are both uncertain due to the inherent slippage between the tracks and the terrain. To deal with the modeling uncertainties and external disturbances, we use the sliding mode control (SMC) approach. A Mobile User Interface (MUI) based on Android operating system. is developed to control the TMR manually or autonomously. By using the MUI the human operator can visualize the information captured from external and internal sensors. Numerical simulations in MATLAB are carried out to verify the performance of the controller as well as validate the robot kinematic model under different configurations.

\section{Keywords}

Tracked Mobile Robots; Robust Control; Uncertain Systems Mobile User Interface; Surveillance Robots; 


\section{Resumo}

Lovon Ramos, Percy Wilianson; Leite, Antonio Candea. Modelagem e Controle de um Robô Móvel com Esteiras para Tarefas de Vigilância. Rio de Janeiro, 2019. 118p. Dissertação de Mestrado - Departamento de Engenharia Elétrica, Pontifícia Universidade Católica do Rio de Janeiro.

Nos últimos anos, os avanços mais recentes em robótica e suas aplicações têm sido usados para reduzir a carga de trabalho e os requisitos de mão-de-obra, melhorando o ambiente, a saúde e a segurança, particularmente nos sistemas de produção agrícola. Robôs autônomos fazem parte de tal inovação tecnológica e os robôs móveis com esteiras, em particular, têm sido amplamente utilizados em campos agrícolas em todo o mundo, já que suas esteiras proporcionam uma grande área de contato em solos úmidos e terrenos irregulares, evitando que o robô fique preso e melhorando a sua mobilidade. Neste trabalho, aborda-se a modelagem e o controle de robôs móveis com esteiras (Tracked Mobiler Robots, TMRs) para executar tarefas de vigilância em campos agrícolas. A metodologia proposta considera que o modelo cinemático do TMR são incertos devido ao escorregamento inerente entre as esteiras e o terreno. Para lidar com as incertezas de modelagem e perturbações externas, utiliza-se uma estratégia de controle robusto baseada na abordagem de modos deslizantes. Uma interface de usuário móvel (Mobile User Interface, MUI) baseada no sistema operacional Android é desenvolvida para controlar o robô movél com esteiras de forma manual ou autônoma. A partir da MUI, o operador humano pode visualizar as informações capturadas de sensores externos e internos. Simulações numéricas em MATLAB são realizadas para verificar o desempenho do controladores, bem como validar o modelo cinemático do robô, em diferentes configurações iniciais.

\section{Palavras-chave}

Robôs Móveis com Esteiras; Controle Robusto; Sistemas Incertos; Interface de Usuário Móvel; Robôs de Vigilância; 


\section{Table of contents}

1 Introduction $\quad 15$

$\begin{array}{lll}1.1 & \text { Motivation } & 16\end{array}$

1.2 Review of the State-of-the-Art 23

1.2.1 Agricultural Mobile Robots 23

1.2.2 Robot Surveillance Problem 26

$\begin{array}{ll}1.2 .3 \text { Human Robot Interfaces } & 29\end{array}$

1.2.3.1 Web Interfaces 30

$\begin{array}{lll}\text { 1.2.3.2 Desktop Interfaces } & 31\end{array}$

1.2.3.3 Mobile Interfaces 31

1.2.4 Modeling Tracked Mobile Robots 33

1.2.5 Controlling the Tracked Mobile Robot 34

1.3 Contribution 35

$\begin{array}{ll}1.4 & \text { Goals and Objectives } \\ 1.4 .1 & 35\end{array}$

1.4.1 Short Term $\quad 35$

1.4.2 Long Term 36

$\begin{array}{ll}1.5 \text { Methodology } & 36\end{array}$

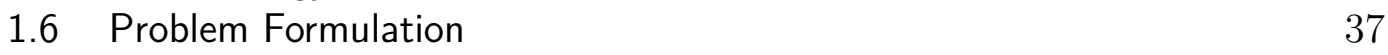

$\begin{array}{lll}1.7 & \text { Text Organization } & 38\end{array}$

2 Modeling and Classical Control Design $\quad 39$

2.1 Description of a Tracked Mobile Robot 39

2.2 Modeling 40

2.2.1 Kinematic Modeling 40

2.3 Regulation Control 43

2.3.1 Cartesian Coordinates based Controller 44

2.3.1.1 Control Design 44

2.3.1.2 Verification $\quad 45$

2.3.2 Polar Coordinates based controller $\quad 51$

2.3.2.1 Control Design $\quad 52$

2.3.2.2 Verification $\quad 55$

3 Robust Control Strategies $\quad 68$

$\begin{array}{lll}3.1 & \text { Chained Form System } & 68\end{array}$

3.1.1 Preliminary Definitions 68

$\begin{array}{ll}\text { 3.1.2 Conversion to Chained Form } & 71\end{array}$

3.1.3 Chained Form for Tracked Mobile Robot $\quad 72$

3.2 Sliding Mode based Controller $\quad 76$

$\begin{array}{lll}3.2 .1 & \text { Verification } & 78\end{array}$

4 Human Robot Interface $\quad \mathbf{8 5}$

4.1 Fundamental Concepts $\quad 85$

4.1.1 Robot Operating System $\quad 85$

$\begin{array}{lll}\text { 4.1.2 ROS Architecture } & 87\end{array}$

$\begin{array}{lll}4.1 .3 \text { RosJava } & 90\end{array}$ 
$\begin{array}{lll}4.2 & \text { System Overview } & 90\end{array}$

$\begin{array}{lll}4.3 & \text { Server } & 91\end{array}$

4.3.1 Publish Sensor Information $\quad 91$

4.3.2 Receive Commands $\quad 92$

$\begin{array}{lll}4.4 \text { Tablet } & 92\end{array}$

$\begin{array}{lll}\text { 4.4.1 Receive Sensor Information } & 93\end{array}$

4.4.2 Send Commands 93

4.5 Nodes in the HRI $\quad 94$

4.5.1 serverActuator $\quad 95$

$\begin{array}{ll}4.5 .2 & \text { listenerSensor } \\ & 95\end{array}$

5 Concluding Remarks $\quad 96$

$\begin{array}{ll}5.1 \text { Discussion } & 96\end{array}$

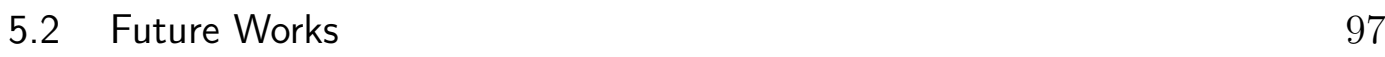

$\begin{array}{ll}\text { Bibliography } & 100\end{array}$

$\begin{array}{ll}\text { A Stability Analysis } & 109\end{array}$

A.1 Proof of Theorem 2.1 109

A.2 Proof of Theorem 2.2 110

A.3 Proof of Theorem $3.10 \quad 112$

B Chained Form for Unicycle Case $\quad 114$ 


\section{List of figures}

$\begin{array}{lll}\text { Figure 1.1 Agricultural Robots Examples } & 16\end{array}$

Figure 1.2 Research articles on Wheeled Mobile Robots for Agriculture.

Figure 1.3 Research articles on robust control for WMR and research articles on SMC in WMR.

Figure 1.4 Research articles for Tracked Mobile Robots.

Figure 1.5 Research articles in Robust Control for Tracked Mobile Robots.

Figure 1.6 A few examples of Tracked Mobile Robots 21

Figure 1.7 Examples of agricultural Mobile Robots 25

$\begin{array}{lll}\text { Figure 1.8 Surveillance Robot example. } & 27\end{array}$

Figure 1.9 Different applications of surveillance robots 28

Figure 1.10 Web Interface Example. 30

Figure 1.11 Desktop Interface Example. 31

Figure 1.12 Mobile Interface Example. 32

Figure 1.13 Problem formulation for a surveillance task in agricultural fields.

Figure 2.1 Tracked Mobile Robot.

Figure 2.2 Tracked Mobile Robot Kinematic Model. 41

Figure 2.3 Regulation Task.

Figure 2.4 Simulation results for Cartesian based controller: First test (I)

Figure 2.5 Simulation results for Cartesian based controller: First test (II).

Figure 2.6 Simulation results for Cartesian based controller: Second test (I)

Figure 2.7 Simulation results for Cartesian based controller: Second test (II).

Figure 2.8 Simulation results for Cartesian based controller: Third test (I).

Figure 2.9 Simulation results for Cartesian based controller: Third test (II).

Figure 2.10 Polar Coordinates Definition.

Figure 2.11 Simulation results for Polar Coordinates based controller: First test (I).

Figure 2.12 Simulation results for Polar Coordinates based controller: First test (II).

Figure 2.13 Simulation results for Polar Coordinates based controller:

First test (III).

Figure 2.14 Simulation results for Polar Coordinates based controller:

Second test (I).

Figure 2.15 Simulation results for Polar Coordinates based controller:

Second test (II). 
Figure 2.16 Simulation results for Polar Coordinates based controller:

Second test (III).

Figure 2.17 Simulation results for Polar Coordinates based controller:

Third test (I).

Figure 2.18 Simulation results for Polar Coordinates based controller:

Third test (II).

Figure 2.19 Simulation results for Polar Coordinates based controller:

Third test (III).

Figure 2.20 Simulation results for Polar Coordinates based controller:

Fourth test (I).

Figure 2.21 Simulation results for Polar Coordinates based controller:

Fourth test (II).

Figure 2.22 Simulation results for Polar Coordinates based controller:

Fourth test (III).

Figure 3.1 Simulation results for Cartesian based controller: First test (I)

Figure 3.2 Simulation results for Cartesian based controller: First test (II).

Figure 3.3 Simulation results for SMC based controller: Second test (I).

Figure 3.4 Simulation results for SMC based controller: Second test (II).

Figure 3.5 Simulation results for SMC based controller: Third test (I).

Figure 3.6 Simulation results for SMC based controller: Third test (II).

Figure 4.1 Communication scheme for ROS 


\section{List of tables}

Table 1.1 Main sensors used for Mobile Robots in Agriculture. 26

Table 1.2 Modeling and Control on TMR 33

Table 2.1 Initial configurations for TMR. 45

Table 2.2 Different configurations on slippage gain of the TMR 46

Table 2.3 Different parameters on gain controllers of Cartesian Controller 48

Table 2.4 Different configurations of gains of Polar Coordinates Controller $\quad 58$

$\begin{array}{lll}\text { Table 3.1 Initial configurations for TMR } & 78\end{array}$

Table 3.2 Different parameters on slippage gain of TMR in SMC 78 


\section{List of Abreviations}

CCTV - Closed Circuit Television

DTFM - Dual Tone Multifrequency

GPS - Global Position System

HRI - Human Robot Interaction

ICR - Instantaneous Center of Rotation

ID - Identification

IMU - Inertial Measurement Unit

IoT - Internet of Things

IP - Internet Protocol

LIDAR - Laser Imaging Detection and Ranging

MUI - Mobile User Interface

OS - Operating System

PIR - Passive Infrared sensor

RGB-D - Red Green Blue-Depth

RHIB -Rigid Hull Inflatable Boat

ROS - Robot Operating System

SAR - Synthetic Aperture Radar

SBSS - Space Based Space Surveillance

SMC - Sliding Mode Control

STA - Super Twisting Algorithm

TMR - Tracked Mobile Robot

UUV - Unmanned Underwater Vehicles

WMR - Wheeled Mobile Robot 
Science without religion is lame, religion without science is blind.

Albert Einstein, Science and religion, 1954. 